\section{Antenatal pelvic floor muscle training has no long-term effect on incidence of SUI}

Antenatal pelvic floor muscle training (PFMT) can prevent onset of postnatal stress urinary incontinence (SUI), but might not continue to prevent SUI several years after pregnancy. Agur et al. have confirmed that the initial benefit of PFMT on incidence of SUI is not maintained over a long period of time, regardless of whether women continue with PFMT.

The authors assessed 164 women who had previously participated in a study on the effect of PFMT on the incidence of SUI. That study involved women diagnosed with bladder-neck mobility during their first pregnancy, who were randomly assigned to either monthly PFMT supervised by a physiotherapist or verbal advice and/or a leaflet on PFMT. At 3 months' follow-up, significantly fewer women in the supervised PFMT group than in the control group had experienced postnatal SUI (19.2\% vs $32.7 \% ; P=0.02)$.

After 8 years, there was no significant difference in the incidence of SUI in the PFMT and control groups $(35.4 \%$ vs $38.8 \%$; $P=0.75)$. Severity of SUI and quality of life were also similar between these groups. Of the women in the supervised PFMT group, $68.4 \%$ continued with PFMT after completion of the initial study and $38.0 \%$ reported that they undertook PFMT at least twice a week; however, the incidence of SUI was similar in those who performed PFMT twice weekly or more and those who performed it less frequently. On multivariate analysis, only presence of SUI at 3 months was predictive of SUI at 8 years.

Original article Agur WI et al. (2008) The long-term effectiveness of antenatal pelvic floor muscle training: eight-year follow up of a randomised controlled trial. BJOG 115: 985-990

\section{Sildenafil improves sexual function in men without erectile dysfunction}

A preliminary study has found that sildenafil can improve sexual performance and satisfaction in men who have not previously reported erectile dysfunction (ED). Sildenafil is an effective treatment for ED, but its effects in men with normal erectile function have been poorly documented.
Gruenwald et al. assessed data from 47 sexually active men (average age $52.1 \pm 10.3$ years) who had no erectile complaints. Initial assessment identified mild ED in 19 men; the remaining 28 did not have ED. In this crossover study, participants received six tablets of sildenafil $50 \mathrm{mg}$ and six placebo tablets, separated by a 2-week washout period, in random order. Sexual function was assessed by quality of sexual life questionnaires. Sildenafil-treated men showed significant improvements in most parameters, and there were no significant differences between those with normal erectile function and those with mild ED. Improved sexual performance and satisfaction in the sildenafil-treated group was attributed to factors such as prolonged erection and reduced intervals between erections. Sildenafil use was also associated with improved self-esteem as a result of improved sexual performance.

Pharmaceutical industry policy states that 'using a prescription drug for a purpose not prescribed is in essence drug abuse'. The present study suggests that men with seemingly normal erectile function or borderline ED can benefit from taking sildenafil, which improves sexual satisfaction for both themselves and their partners. These data could influence the debate over whether sildenafil should be available without prescription.

Original article Gruenwald I et al. (2008) Effect of sildenafil on middle-aged sexually active males with no erectile complaints: a randomized placebo-controlled double-blind study. Eur Urol [doi:10.1016/j.eururo.2008.04.048]

\section{RNA expression profiles might be useful predictors of prostate cancer recurrence}

PSA doubling time does not indicate which men with biochemical recurrence after radical retropubic prostatectomy (RRP) would benefit from additional clinical interventions. Nakagawa and colleagues, therefore, identified a panel of genes whose RNA expression is likely to be altered in patients with prostate cancer progression, and conducted a nested case-control study to test the panel's ability to predict outcome.

Cases were 213 men who developed systemic progression within 5 years after PSA recurrence, and age-matched controls were 